\title{
ECCENTRICITY EFFECTS UPON THE FLOW FIELD INSIDE A WHIRLING ANNULAR SEAL
}

\author{
Gerald L. Morrison, Robert E. DeOtte, Jr., \\ Purandar G. Das, and H. Davis Thames \\ Texas A\&M University \\ College Station, Texas
}

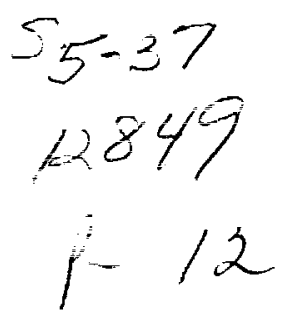

\begin{abstract}
The flow field inside a whirling annular seal operating at a Reynolds number of 24,000 and a Taylor number of 6,600 has been measured using a 3-D laser Doppler anemometer system. Two eccentricity ratios were considered, 0.10 and 0.50 . The seal has a diameter of $164 \mathrm{~mm}$, is 37.3 $\mathrm{mm}$ long and has a clearance of $1.27 \mathrm{~mm}$. The rotor was mounted eccentrically on the shaft such that the whirl ratio is 1.0 and the rotor follows a circular orbit. The mean axial velocity is not uniform around the circumference of the seal; near the inlet a region characterized by high velocity is evident on the pressure side of the seal, but the velocity is relatively uniform by the center plane of the seal. By the exit, another region of high axial velocity has developed, this time on the suction side of the seal. The magnitude and azimuthal distance of the migration increased with increasing whirl amplitude (eccentricity). Throughout the seal length the azimuthal mean velocity varied inversely with the mean axial velocity. Increasing the whirl amplitude did not increase the magnitude of the azimuthal velocity at the seal exit.
\end{abstract}

\section{NOMENCLATURE}

$\begin{array}{ll}\text { A } & \text { Leakage area } \\ \mathrm{c} & \text { Mean clearance } \\ \mathrm{d} & \text { Rotor diameter } \\ \mathrm{e} & \text { Eccentricity } \\ \mathrm{L} & \text { Length of the seal } \\ \mathrm{Q} & \text { Leakage flow rate } \\ \mathrm{r} & \text { Radial distance from stator centerline } \\ \mathrm{Re} & \text { Reynolds number }=2 \mathrm{Uc} / \nu \\ \mathrm{Ta} & \text { Taylor number }=\frac{c W_{s h}}{v} \sqrt{\frac{2 c}{d}} \\ \mathrm{U} & \text { Axial mean velocity } \\ \mathrm{U}_{\text {ave }} & \text { Bulk mean velocity }=\mathrm{Q} / \mathrm{A} \\ \mathrm{V} & \text { Radial mean velocity }\end{array}$

$\begin{array}{ll}\frac{\mathrm{W}}{\boldsymbol{W}^{\prime} \boldsymbol{u}} & \text { Tangential mean velocity } \\ \mathrm{W}_{\mathrm{sh}} & \text { Time averaged Reynolds stress } \\ \mathrm{Z} & \text { Rotor surface speed } \\ \epsilon & \text { Distance from seal inlet } \\ \theta & \text { Accentricity ratio, e/c } \\ \mu & \text { Fluid absolute viscosity } \\ \nu & \text { Kinematic viscosity } \\ \rho & \text { Fluid density } \\ \omega & \text { Whirl ratio, } \omega_{\mathrm{p}} / \omega_{\mathrm{r}} \\ \omega_{\mathrm{p}} & \text { Rotor precession speed } \\ \omega_{\mathrm{r}} & \text { Rotor speed }\end{array}$

\section{INTRODUCTION}

A seal, by definition, separates regions of high and low pressure fluids. In some cases, the desire is to prevent leakage, but that is not the general situation. Usually the seals in rotating machinery. such as pumps and compressors, are designed to control but not eliminate the leakage flow which may cool and enhance operating stability by improving vibration damping. Seal design involves balancing considerations of machine efficiency, which is generally higher with lower leakage, with operational stability.

In many applications, the shaft itself plays the part of the seal. The seal clearance is usually less than a tenth of a millimeter. Obtaining accurate measurements in these small regions is, at best, difficult. As a consequence, the database of velocity and turbulence measurements is

\footnotetext{
'Presently a Senior Engineer at BW/IP International, Inc. Pump Division, Vernon, CA.
} 
very small. Further complicating matters is the turbulent flow found under normal operating conditions. The lack of detailed flow field information hinders the development of accurate seal performance analyses.

Research in this area has been mainly devoted to studying leakage and rotordynamic coefficients. Though quite important for gaining an insight into the behavior of these seals, they alone do not present a complete picture. Velocity measurements have been made in models with clearance ratios (seal clearance to seal diameter) much larger than those found in actual applications. Turbulence measurements, however, are much scarcer. Most of the data available is for seals operating under static conditions and does not provide the knowledge of both the mean velocities and the turbulent quantities necessary for numerical modelling of the flow fields.

Although ideal operating conditions are assumed for seal design and modelling purposes, this is often not the case. During machine operation, shafts become misaligned resulting in the seal spinning eccentric to the center of rotation thus altering the dynamic forces. The flow in such an eccentric seal is different from that in a concentric seal. To provide data applicable to such routine situations, the rotors/seals studied were deliberately spun off center.

One of the important objectives of this study is to help build a database of flow measurements for annular seals over a range of operating conditions. Earlier work done at this lab, Thames[1992], focussed on flow through an eccentric seal with a 0.5 eccentricity ratio. That study represents an extreme case of misalignment. This work compares an annular seal, operating with a 0.1 eccentricity ratio, to the 0.5 eccentricity ratio case operating at the same conditions.

\section{Literature Review}

Nelson and Nguyen[1987] working at Texas A \& M University, studied the effects of eccentricity on rotordynamic coefficients. They presented a new analysis procedure that solved for flow variables in an eccentric annular seal. The governing equations were based on a turbulent bulk flow model and Moody's friction factor equation. Comparisons with experimental data led them to conclude that this method was more accurate in predicting the rotordynamic coefficients than one which used a finite element analysis procedure. The accuracy of the procedure could be improved significantly if a more accurate friction factor formula for annular seals was found.

Chen and Jackson[1987] studied the influence of eccentricity and misalignment on the performance of high-pressure annular seals. Using existing concentric seal theories they developed a method for the calculation of both seal leakage and dynamic coefficients for short seals with large eccentricities and/or misalignment of the shaft. The Space Shuttle Main Engine High Pressure Fuel Turbopump pump interstage seal was used for calculating the leakage coefficients. From the ensuing predictions they concluded that the effect of eccentricity on the leakage rate was higher in the case of laminar flow than when the flow was turbulent. They also found that as the eccentricity/misalignment ratio (inlet versus outlet) increased the mass flow leakage decreased due to an increase in the passage blockage.

Allaire, et al.[1978], while studying the dynamics of short eccentric plain seals with high axial Reynold's numbers, saw a "Bernoulli" effect in eccentric seals. A bulk flow theory developed by Hirs[1973] was used in the analysis. They found that for a given axial pressure drop across the seal, the axial fluid velocity is greatest in the maximum clearance portion of the seal and least in the minimum clearance portion of the seal. The pressure distribution around the shaft was found to be inversely related to the velocity distribution. This pressure distribution induced a lydrodynamic force which tended to center the shaft. At low Reynold's numbers the pressure differences in the seal were small and mainly due 10 friction effects. At high Reynold's numbers the friction effects were small and the pressure was constant around and along the seal. In conclusion. they stated that the load capacity of the seal was maximized for a given value of the axial Reynold's number, when the Bernoulli effect was the greatest. At very high Reynold's 

numbers, large head losses occurred at the entrance to the seal which induced a constant pressure
and diminished the load capacity of the seal.

Tam and et al.[1988] numerically calculated and compared leakage rates and dynamic of the foeficients for eccentric annular seals. The numerical model was based on a conservative form of the three dimensional Navier-Stokes equations. The analytical model was based on "lumped" and a high pressure characteristics of the seal were summarized as strong swirl, high viscosity turbulence closure drop associated with significant wall roughness and small clearances. The was chosen because obtained with the zero-equation Prandtl mixing length model. This model clearance. The eddy vis simplicity and the fact that the mixing length can be estimated by the was obtained using the SIMPLEST was used to compute the Reynold's stresses. The solution specified pressure at the SIMPLEST algorithm. The boundary conditions employed included: velocity and rotor precessional speed shear stresses on the surfaces of the rotip velocity condition at the rotor and stator walls, fluid circumferential direction. A three dimetor and stator, and cyclic boundary conditions in the dimensions $12 \times 6 \times 16$ was employed. along the seal, as well as the presed. The results showed significant changes in the fluid forces pressure drops. It was found that at large axial secondary flow zones were intenat at lower rotational speed and higher perturbation speed, the enhancing the stability of the retified. This, in turn, lowered the average circumferential velocity, clearance zone and of the rotor. A secondary flow zone occurred just in front of the minimum inlet end of the same speed as the shaft. Preswirl, introduced at the also studied. The fluiduenced the average circumferential velocity. Fluid injection effects were Injection in the fir was injected radially, both with and against the direction of rotation. observed when the injection rotation increased the leakage rate, whereas, a drop of about $7 \%$ was leakage rate was found to be most influnst the direction of rotation. In conclusion, the average perturbation speed on leakage rate was med by the axial pressure drop. The effect of rotation and assumed.

The static and dynamic characteristics of plain annular applications, considering the effect They studied a tapered seal mainly with respect to cryogenic variable fluid properties. Assuming swirl, axial acceleration effect of liquid at the inlet and inertial forces negligible they coming that pressure, viscous, and turbulent stresses rendered the fluid obtained good agreement. constant fluid properties. In conclusion they observed lower leakage rates for a straight seal with leakage was initially found to increasing $\mathrm{L} / \mathrm{D}$ ratio's of up to a value of 0.2 . For tapered seals the thereafter.

Kanemori and Iwatsubo [1989] conducted an acting on a long annular seal. They founducted an experimental study of the dynamic forces which does not make any considerations for their results agreed with Fritz's [1970] theory, force in the seal depended on the whirl for axial flow. They suggested that the fluid reaction velocity acted as a de-stabilizing force.

Johnson [1989] and Morrison, et al[1991a] investigated the flow filed inside centered and statically eccentric annular seals using a 3-D Laser Doppler velocimeter. The seal, with a clearance of $0.0127 \mathrm{~mm}$, was operated at a Reynolds number of 27,000 and a Taylor number of 6,600. The mean velocity vector and the entire Reynolds stress tensor at four angular positions intermediate locations corresponding to the minimum clearance, maximum clearance and two considered included no pre-swirl, backard the entire length of the seal. The different flow cases swirl case the profile of azimuthal velocity appeared to be reasonably well developed for all 
clearances whereas for the no pre-swirl and backward pre-swirl cases, substantial flow development occurred for each clearance. Pre-swirl does have a pronounced effect, although it is not significantly greater for the statically eccentric than the concentric configuration.

Thames [1992] measured the flow field inside a whirling annular seal $(\epsilon=0.5)$ using a 3-D Laser Doppler Velocimeter with phase averaging. Two axial flow conditions $(\operatorname{Re}=12,000$ and $\operatorname{Re}=24,000$ ) were considered. Velocity and Reynolds stress distributions were presented for both flow conditions. The existence of a region of high axial momentum, which at the inlet is on the high pressure side of the seal and migrates to the suction side at the exit of the seal, was identified.

\section{OBJECTIVES}

Most studies of seals have been empirical or numerical. The time averaged Navier-Stokes equations were solved by finite difference techniques using a $k-\epsilon$ turbulence closure model. Although the numerical solutions predict the flow field, experimental data is required for verification. This study was undertaken to measure the flow field through eccentrically whirling annular seals. Measurements made were of the instantaneous velocity components in three independent directions. From these, the mean flow velocity in the axial, radial and tangential directions were calculated along with the turbulent kinetic energy and the phase averaged Reynolds stresses.

\section{FACILITIES AND INSTRUMENTATION}

The experiments were performed in the seal test facility of the Turbomachinery Laboratory which has been described previously by Morrison, et al.[1991a]. The test section (Figure 1) consists of a $50.8 \mathrm{~mm}$ diameter overhung shaft which is rotated by a variable speed electric motor. The rotor is manufactured of acrylic and is mounted onto the shaft via a bushing. For the present study, mounting bushings were made so that the inner hole was eccentric with the outer surface such that eccentricity ratios of 0.1 and 0.5 were obtained. This mounting results in the rotor whirling at the same speed as the shaft, i.e. a whirl ratio of 1.0. The stainless steel stator is mounted concentric with the overhung shaft. For this set of experiments, the internal contoured plug in the plenum (as used by Morrison[1991a]) was not installed leaving the face of the rotor completely exposed to the plenum.

Water is provided to the test section from a $19 \mathrm{~m}^{3}$ storage tank via a centrifugal pump, throttling valve, and turbine meter. The throttling valve is adjusted to produce the required flowrate through the seal. The water exiting the test section is returned to the storage tank. During the tests, the water temperature remained between

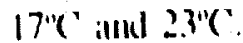

The velocily field inside the annular seal is measured using

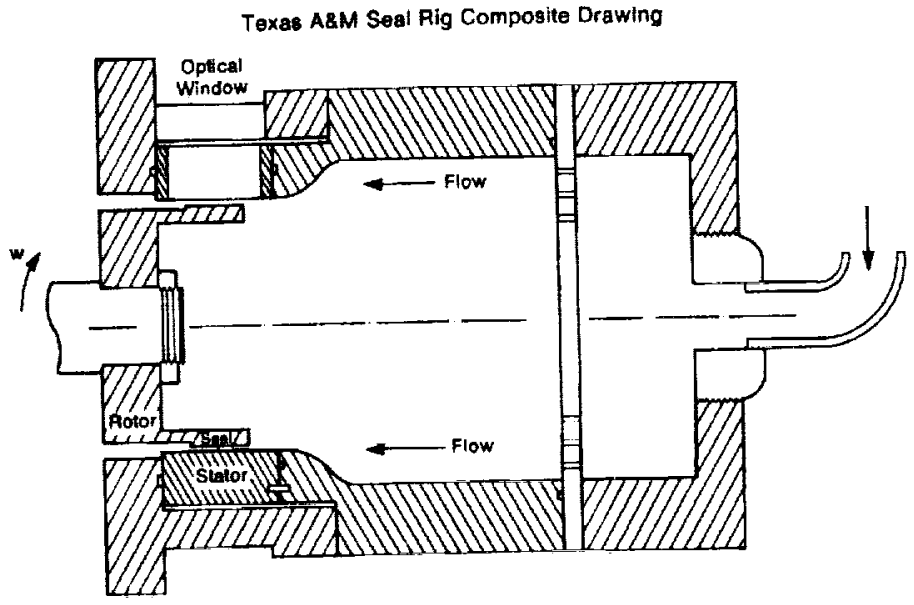

Figure 1. Seal Test Facility. a 3-D Laser Doppler Anemometer (LDA) system. This system has been described in detail in Morrison, et al.[1991a] and 
Johnson[1989]. The 3-D LDA system uses three colors of laser light to measure three instantaneous non-orthogonal velocity components at one location in space. The composite probe colume is $0.025 \times 0.025 \times 0.102 \mathrm{~mm}$. The three instantaneous non-orthogonal velocity componcents are used to calculate lhe instantineous velocity vector in the scial coordinales. $\Lambda$ rotary encoding system was used to tag each instantaneous velocity measurement with the angle of the rotor when the measurement was obtained. For each spatial location, 90,000 individual instantaneous velocity measurements were made which were then phase averaged (into $18^{\circ}$ increments) to determine how the mean velocity varies with rotor position. The phase averaged Reynolds stress tensor was also calculated from the data; however, because of space limitations, only the axial velocity variance will be presented in this paper. Measurements were made across the clearance at 20 different axial locations from slightly upstream of the rotor to the exit plane of the rotor. A complete listing of the data for all angles and axial locations is contained in Thames[1992] and Das[1993]. Morrison, et al[1991b], Thames[1992], and DeOtte, et al[1992] have discussed all the details concerning the accuracy of the measurements made with this system. For the present study, the McLaughlin-Tiederman[1973] velocity bias correction technique was used. The uncertainty of the measurements is as follows: $U \pm 0.115 \mathrm{~m} / \mathrm{s}, \mathrm{V} \pm 0.432 \mathrm{~m} / \mathrm{s}, \mathrm{W} \pm$ $0.095 \mathrm{~m} / \mathrm{s}$, and $\overline{u_{x}^{\prime} u_{x}^{\top}} \pm 0.013 \mathrm{~m}^{2} / \mathrm{s}^{2}$. Unexpanded polystyrene spheres $(6 \mu \mathrm{m}$ in diameter $)$ were used as the seed for the LDA system which possesses a frequency response in excess of $44 \mathrm{kHz}$
in the water.

\section{RESULTS}

Axial Velocities

Contour plots of axial mean velocity at axial positions of $\mathrm{Z} / \mathrm{L}=0.00,0.21,0.50 .0 .77$, and 1.00 in Figure 2 compare the two eccentricity ratios of 0.1 and 0.5 . The minimum clearance is at the top of each plot and the rotor is both rotating and whirling in a clockwise direction, i.e. the pressure side of the seal is on the right $\left(0 \leq \theta \leq 180^{\circ}\right)$. For reference, the bulk average velocity $(\mathrm{Q} / \mathrm{A})$ is $7.4 \mathrm{~m} / \mathrm{s}$. For $\epsilon=0.1$, at the entrance, the mean axial velocity possesses a saddle back profile (velocities higher near the rotor and stator than in the center of the clearance). Such profiles are characteristic of flow encountering an abrupt change in area such as flow through an orifice. The saddle back velocity profile is still present at $\mathrm{Z} / \mathrm{L}=0.21$ but has decreased in severity and by the center of the seal $(Z / L=0.5)$ the saddle back has disappeared. Turbulence generated by shear stresses at the wall diffuses fluctuating momentum radially across the seal which reduces gradients in the mean axial velocity profile. Previous work by Morrison, et al.[1991a] for a non-whirling seal operating at the same Reynolds and Taylor numbers did not show the saddle back profile but in that case a contoured plug was present in the upstream plenum. The plug in conjunction with the stator contour created a nozzle which gently guided the flow into the seal thus eliminating the saddle back profile. At the higher eccentricity ratio $(\epsilon=0.5)$ large disturbances caused by the whirling seal overwhelms the influence of the sudden contraction and the saddle back does not occur. In both cases, the azimuthal location of maximum axial velocity is not at the location of maximum clearance at the seal inlet but is located on the pressure side of the seal. The shift away from the maximum clearance location is more pronounced for the larger eccentricity case. Both cases also exhibit the interesting behavior of the migration of the high axial velocity region from the pressure side of the seal at the inlet to the suction side at the exi the maximum clearance at the $X / L=0.50$. The inlet to the suction side at the exit passing through from the entrance to the center of tangential distribution. From the seal where the axial velocity reaches its most uniform region of higher velocity develops ate plane to the exit, the foncentrated such that a distinct larger eccentricity. It is hypoths at the exit plane. All of these effects were accentuated by the the location of the maximumesized that the fluid entering the seal preferentially heads towards fluid does not reach the clearance. However, since the location is continually changing, the 
towards the azimuthal location of maximum clearance or accelerate and enter the smaller clearance. Since a smaller acceleration is required in the axial direction than in the tangential direction, the required pressure gradient is smaller and the fluid accelerates axially. Discussion about the migration of the axial momentum to the suction side will be delayed until the azimuthal velocities have been presented.

\section{Radial Velocities}

Figure 3 presents contours of the radial mean velocity. The radial velocities are maximum in the inlet plane as the flow adjusts from the large plenum cross sectional area to the substantially smaller clearance area. The radial velocities become negligible past $\mathrm{Z} / \mathrm{L}=0.21$ as the flow adjusts to the small channel clearance. The flow sweeps into the seal from the stator wall resulting in the large negative velocities at the inlet plane will reach maximum values close to the region where the axial velocities are largest. The whirling of the seal tends to block the flow from entering from the plenum region upstream of the rotor face.

\section{Azimuthal Velocities}

The mean azimuthal velocities are shown in Figure 4. Please note that the rotor surface speed is $30.9 \mathrm{~m} / \mathrm{s}$. The contour levels were adjusted to show more clearly the fluid velocity. The inlet plane contours illustrate that the rotor induces a significant amount of preswirl into the fluid by the time it reaches the seal inlet. This is more pronounced for the $\epsilon=0.5$ case. The blockage of the incoming fluid due to the large eccentricity results is the higher levels of swirl for this case. For the $\epsilon=0.1$ condition, the azimuthal mean velocity has a saddle back type profile, as did the axial velocity, which is roughly inversely proportional to the axial mean velocity. That is, when the axial velocity is high, the azimuthal velocity is low and when the axial velocity is low the azimuthal velocity is high. This inverse proportionality is also seen at the inlet for $\epsilon=0.5$ with the azimuthal velocities being larger on the suction side and the axial velocities larger on the pressure side. This may be a simple residence time effect with the slower axial velocity increasing the residence time of the fluid which allows the tangential shear produced by the rotor to impart more azimuthal velocity to the fluid. For the $\epsilon=0.5$ case, the inverse proportionality of the axial and azimuthal velocity components continues to the exit plane where the largest azimuthal velocities have migrated to the pressure side and the largest axial velocities are on the suction side. This migration of the maximum axial momentum flux from the pressure side to the suction side is caused over the first half of the seal $(0 \leq \mathrm{Z} / \mathrm{L} \leq 0.5)$ by the fluid expanding from the pressure side with its small clearance to the location of maximum clearance. This is most likely due to an azimuthal pressure gradient. However, it is the azimuthal velocity which becomes increasingly larger on the pressure side pumping additional fluid from the pressure side to the suction side as the flow continues further downstream. The same effect is seen in for the $\epsilon=0.1$ case but to a lesser extent since the effects of the whirl are decreased by the decreased eccentricity. The mean azimuthal velocity approached $10 \mathrm{~m} / \mathrm{s}$ ( $32 \%$ of the rotor surface speed) at the exit of the seal with very little azimuthal variation for the $\epsilon=0.1$ case but a much greater azimuthal variation for the $\epsilon=0.5$ case. This is larger than the $20 \%$ measured by Morrison, et al.[1991a] for a non-whirling annular seal. However, Morrison, et al. also observed inlet azimuthal velocity values of less than $10 \%$ of the rotor speed compared to the $13 \%$ to $30 \%$ observed in the present case. The elimination of the centerline plug in the plenum resulted in significantly higher preswirl for the present study. The fact that the same levels of swirl are present at the seal exit for both the $\epsilon=0.1$ and 0.5 cases show that increased eccentricity (whirl amplitude) does not increase the level of swirl induced by the rotor. 


\section{Axial Velocity Variance}

The axial velocity variances shown in Figure 5 illustrate that the majority of turbulence production occurs during the first half $(0 \leq \mathrm{Z} / \mathrm{L} \leq 0.5)$ of the seal lengll. Initially the largest turbulence levels are located near the upstream lip of the rotor where the accelerating fluid comes in contact with the rotor surface resulting in large radial and axial velocity gradients. The shear layer generates large levels of vorticity and produces turbulence. The turbulence production continues along the first quarter of the seal length with the turbulence levels remaining high (exceeding turbulence intensities $\left[\sqrt{\overline{u^{\prime} u^{\prime}}} / U_{\text {ave }}\right]$ of 50\%) and spreading across the seal clearance. The turbulence level for $\epsilon=0.1$ is significantly larger at $Z / L=0.219$ than for $\epsilon=0.5$. This is a result of the saddle back velocity profiles present only for $\epsilon=0.1$. The saddle back profiles posses larger regions of mean velocity gradient which in turn generate more turbulence. From 0.21 to $0.50 \mathrm{Z} / \mathrm{L}$ the turbulence level decreases as the mean flow adapts to the small clearance. The mean velocity gradients (and vorticity) are smaller decreasing the turbulence production. From $\mathrm{Z} / \mathrm{L}=0.5$ to the exit of the seal the turbulence levels remain relatively constant with only a slight increase near the rotor surface at the exit plane which is caused by the expansion into the region
downstream of the seal.

\section{CONCLUSIONS}

The complex three dimensionality of the flow through whirling annular seals precludes the use of such quasi-steady state models as that of a statically eccentric seal. Such would predict the maximum axial velocity to be located at the maximum clearance over the entire length of the seal. For both the $\epsilon=0.1$ and 0.5 cases this is not true but instead the maximum axial velocity is on the pressure side of the seal at the inlet and migrates to the suction side at the exit. The larger eccentricity amplified the behavior. The larger eccentricity greatly modified the inlet velocity profiles: the saddle back profiles were eliminated by the rotating blockage effect. The mean azimuthal velocity approached $10 \mathrm{~m} / \mathrm{s}$ ( $32 \%$ of the rotor surface speed) at the exit of the seal. For the $\epsilon=0.1$ case azimuthal variation was small but is was much greater for the $\epsilon=0.5$ case. The mean radial velocities were largest at the entrance plane due to the flow contracting into the clearance from the plenum but diminished in magnitude rapidly past the entrance. Maximum turbulence production and turbulence levels were present over the first $25 \%$ of the seal length with turbulence production and dissipation coming into equilibrium by the center of the seal. Turbulence intensities exceeded $50 \%\left[\sqrt{\overline{u^{\prime} u^{\prime}}} / U_{\text {ave }}\right]$ in the entrance region. Overall the flowfield is very complex and three dimensional suggesting very complex surface forces.

\section{REFERENCES}

Allaire, C.C., Lee, E.J. and Gunter, P.E., 1978, "Dynamics of short eccentric plain seals with high axial Reynolds number.", J. Space. \& Rockets, Vol. 15, No. 6, pp. 341-347.

Chen, W.C. and Jackson, E.D., 1987, "A general theory for eccentric and misalignment effects in high-pressure annular seals.", ASLE Transactions, Vol. 30, No. 3, p. 293-301.

Childs, D.W. and Scharrer J.K., 1986, "An Iwatsubo-based solution for labyrinth seals: comparison to experimental results.", Transactions ASME Journal of Engineering. Gas Turbines
\& Power, Vol. 2, p. 325-331.

Das, P.G., 1993, "3-D LDV Measurements of Eccentric Annular and Labyrinth Seals," Master of Science Thesis, Texas A\&M University.

DeOtte, Jr. R.E., Morrison, G.L., and Wiedner, B.G., 1992, "Considerations on the Velocity Bias in Laser Doppler Velocimetry, Presented at the Sixth International Symposium on Applications of Laser Techniques to Fluid Mechanics, Istituto Superior Tecnico, Av. Rovisco Pais, 
Fritz, R.J., 1070, "The Effects of an Annular Fluid on the Vibrations of a Long Rotor, Part2-Test.", Trans. ASME Journal Basic Engineering., p. 930.

Hirs, G.G., 1973, "Bulk Flow Lubricant Theory for Turbulence in Lubricant Films," ASME Journal of Lubricant Technology, Vol. 110, pp. 137-146.

Johnson, M.C., 1989, "Development of a 3-D Laser Doppler Anemometry System:With Measurements in Annular and Labyrinth Seals.", Ph.D. Dissertation, Texas A\&M University.

Kanemori, Y., and Iwatsubo, T., 1989, "Experimental Study of Dynamical Characteristics of a Long Annular Seal," JSME International Journal Series II, Vol. 32, pp. 218-224.

Morrison. G. M., Johnson, M.C., and Tatterson, G.B., 1991, "Three Dimensional Laser Doppler Measurements in an Annular Seal," ASME Journal of Tribolgy, Vol. 113, pp. 390-396.

McLaughlin, D.K. and Tiederman, W.G., 1973, "Biasing Correction for Individual Realization of Laser Anemometer Turbulent Flows", The Physics of Fluids, Vol. 16, pp. 20222088.

Morrison. G.L., Johnson, M.C., and Tatterson, G.B., 1991a, "Three Dimensional Laser Doppler Measurements in an Annular Seal," ASME Journal of Tribolgy, Vol. 32, pp. 390-396.

Morrison, G.L., Johnson, M.C., Swan, D.H., and DeOtte, Jr., R.E., 1991b. "Advantages of Orthogonal and Non-orthogonal Three-Dimensional Anemometer Systems," Flow Measurement and Instrumentation, Vol. 2, pp. 89-97.

Nelson, C.C. and Nguyen, D.T., 1987, "Analysis of Eccentric Annular Incompressible Seals: Part 1. A New Solution Using Fast Fourier Transforms For Determining Hydrodynamic Force." New York, U.S.A., American Society of Mechanical Engineers. ASME paper No. 87Trib-52.

Simon F, and Freene, J, 1989, "Static and Dynamic Characteristics of Turbulent Annular Eccentric Seals: Effect of Convergent-Tapered Geometry and Variable Fluid Properties," $A S M E$ Journal of Tribology, Vol. 111, pp. 378-385.

Tam, L.T. Przekwas, A.J., Muszynska, A., Hendricks, R.C., Braun, M.J., and Mullen, R.L.. 1988. " Numerical and Analytical Study of Fluid Dynamic Forces in Seals and Bearings," Joumal of Vibration, Acoustics, Stress, and Reliability in Design, Vol. 110, pp. 315-325.

Thanes, H.D., 1992. "Three Dimensional Laser Doppler Measurements of an Annular Seal." Master of Science Thesis, Texas A\&M University. 

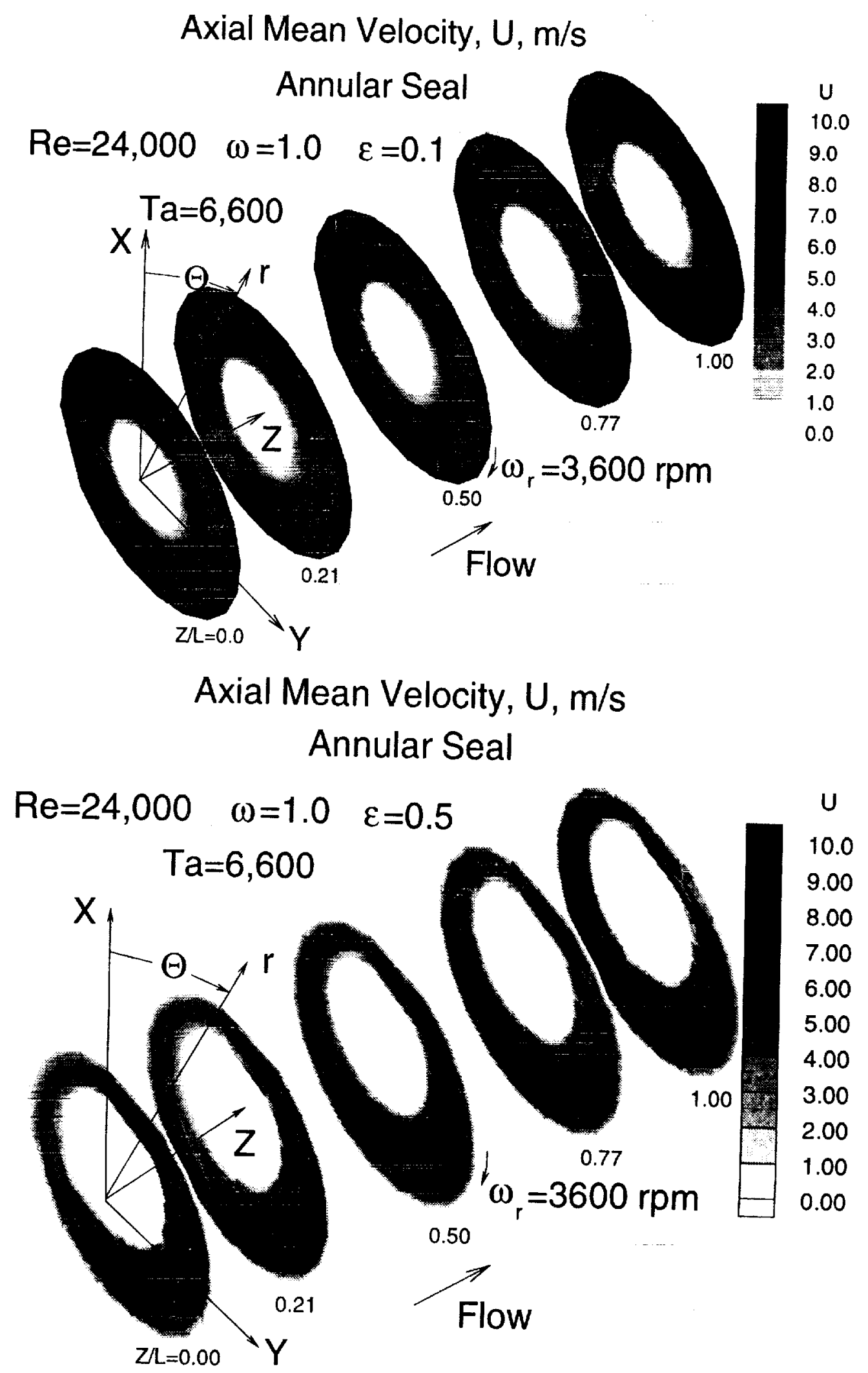

Figure 2. Axial Mean Velocity Contours 
Radial Mean Velocity, V, m/s

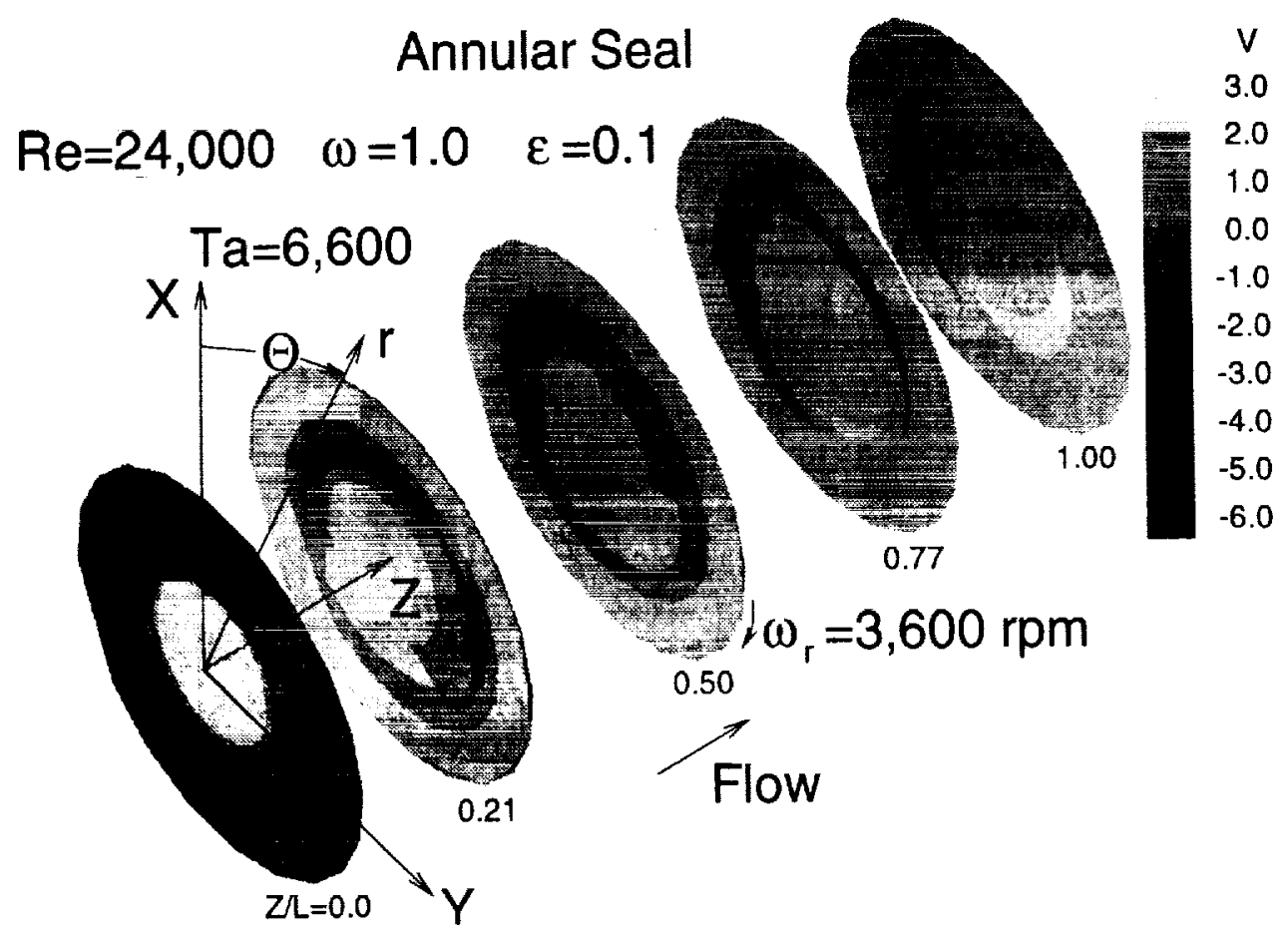

Radial Mean Velocity, V, m/s Annular Seal

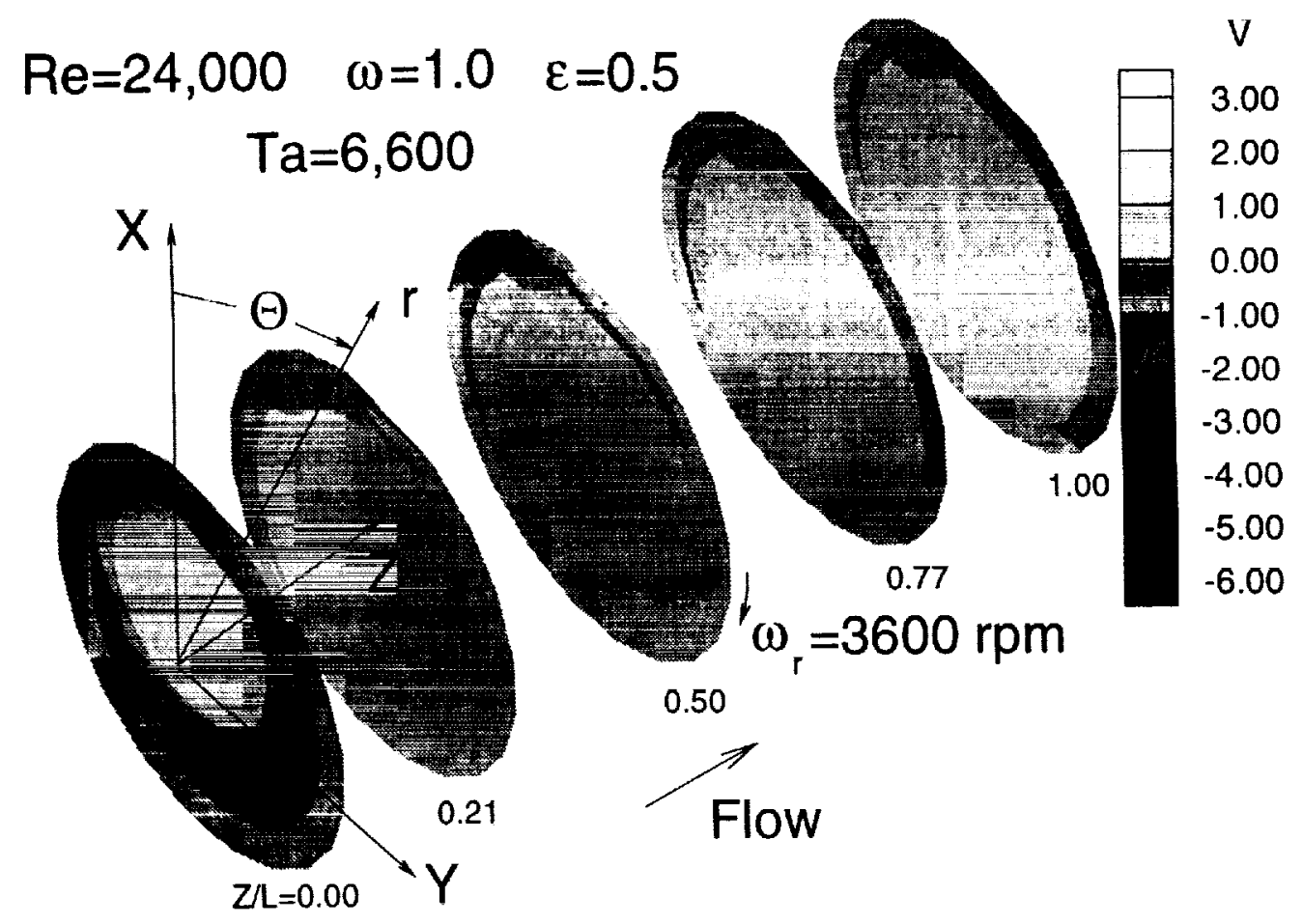

Figure 3. Radial Mean Velocity Contours 
Azimuthal Mean Velocity, W, m/s

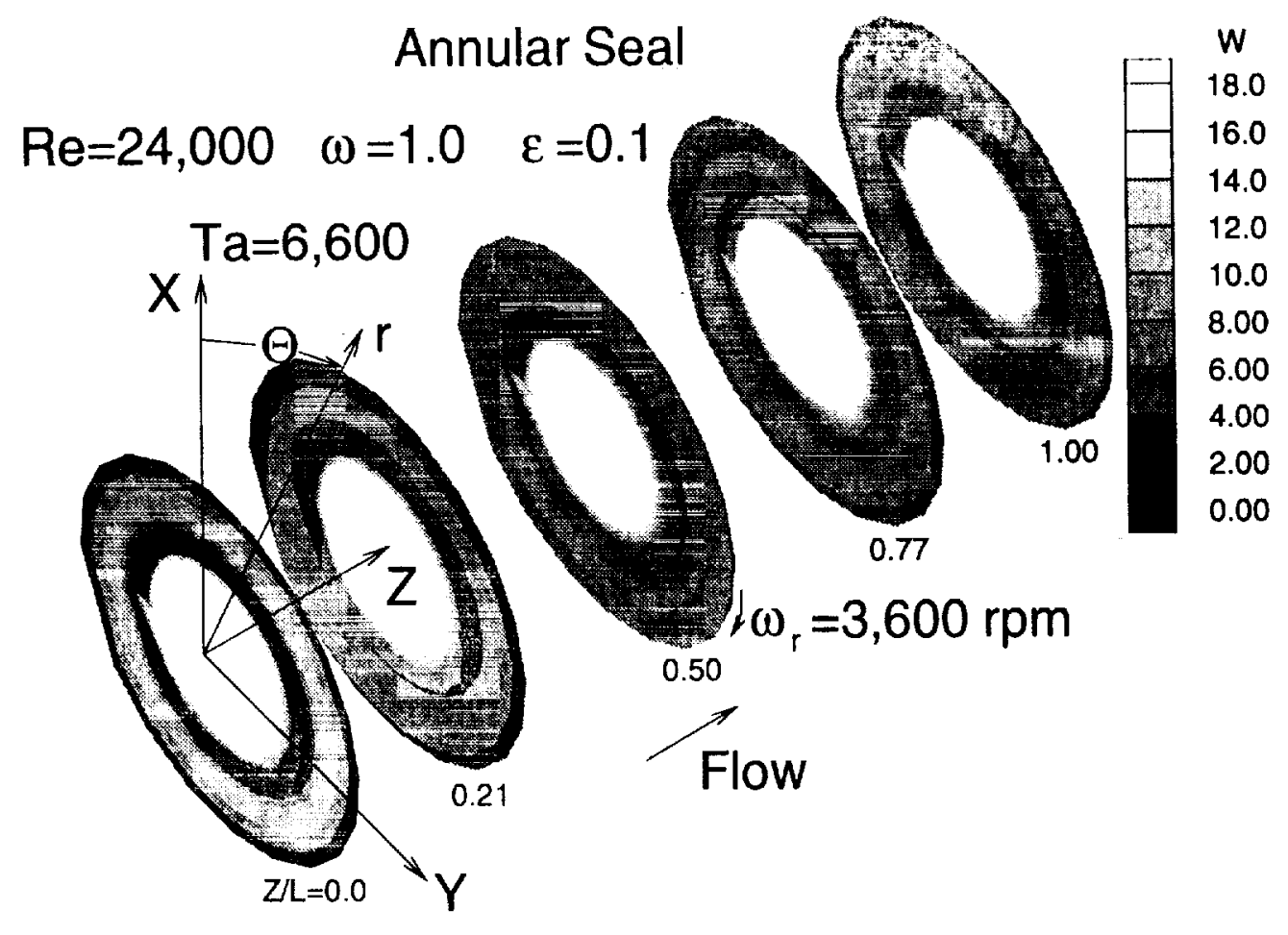

Azimuthal Mean Velocity, W, m/s Annular Seal

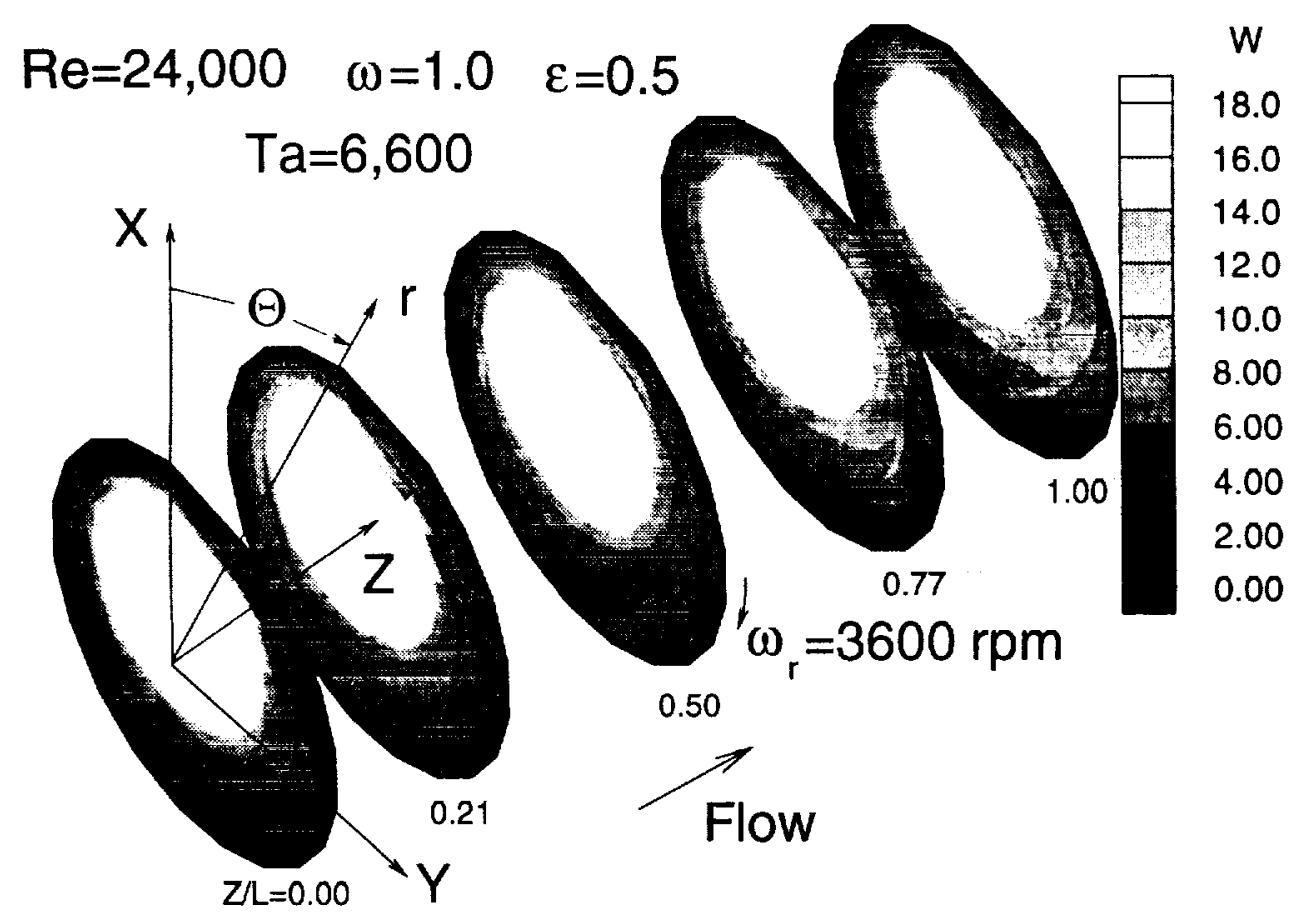

Figure 4. Azimuthal Mean Velocity Contours 
Axial Velocity Variance, uu, $\mathrm{m}^{2} / \mathrm{s}^{2}$

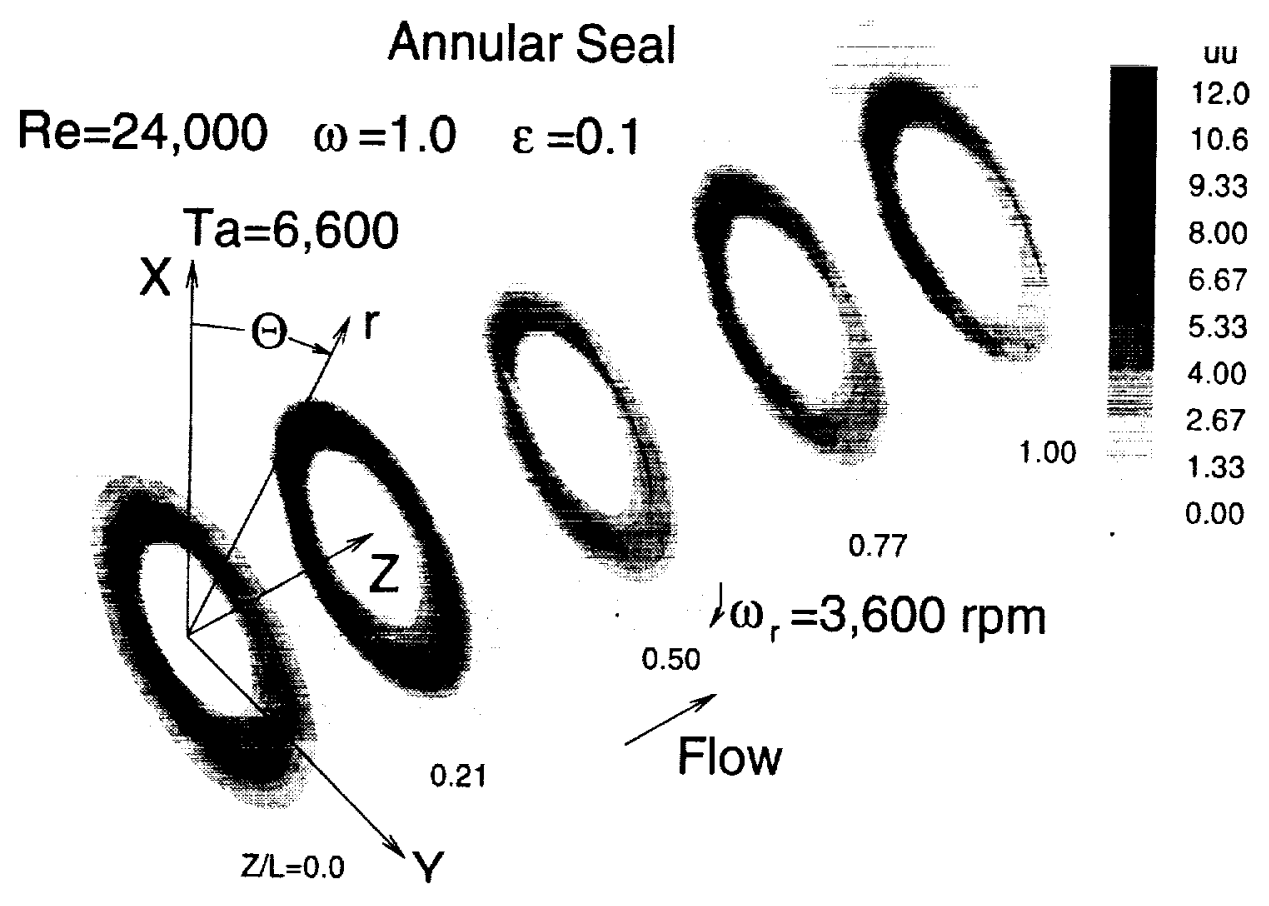

Axial Velocity Variance, u'u', $\mathrm{m}^{2} / \mathrm{s}^{2}$
Annular Seal

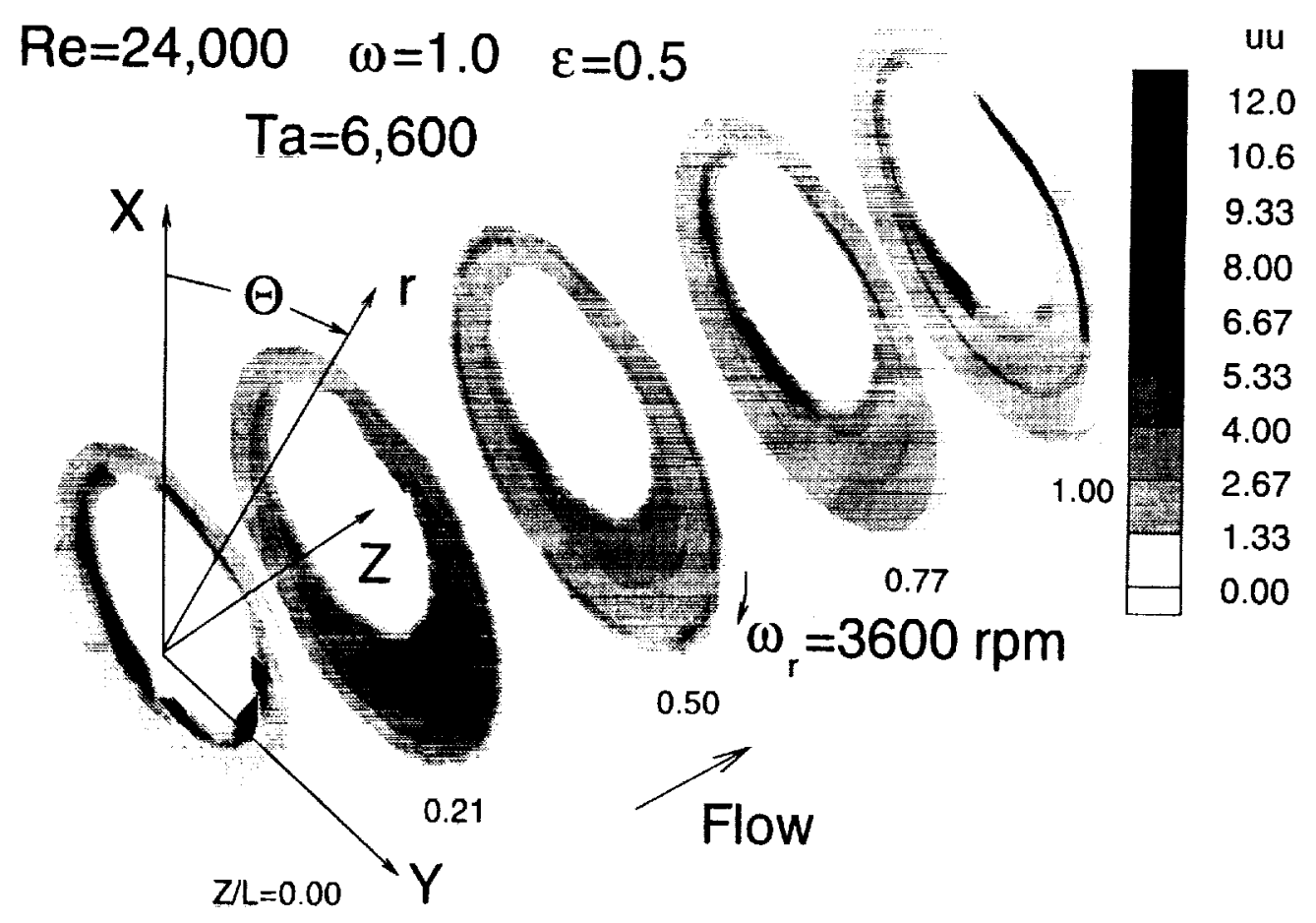

Figure 5. Axial Velocity Variance Contours 